DOI: $10.12957 /$ demetra.2017.26426

\title{
Detecção dos genes mecA e seh de Staphylococcus sp. isolados de amostras de alimentos, superfícies e utensílios de uma cozinha industrial do Rio de Janeiro
}

\author{
Detection of mecA and seh genes and password Staphylococcus sp. isolated from food \\ samples, surfaces and utensils of an industrial kitchen of Rio de Janeiro
}

Paula Lisbôa Nascimento ${ }^{1}$

Juan de Oliveira Martinezl

1 Instituto Federal de Educação, Ciência e

Tecnologia do Rio de Janeiro - campus Rio de

Janeiro. Rio de Janeiro-RJ, Brasil.

Correspondência / Correspondence

Paula Lisbôa Nascimento

lisboa.paula33@gmail.com

\section{Resumo}

Os Staphylococcus são micro-organismos cada vez mais associados a intoxicações alimentares. Este trabalho teve como objetivo determinar a prevalência e detectar fatores de virulência e resistência de Staphylococcus spp. isolados de amostras de alimentos, dos utensílios e superfícies de manipulação de uma cozinha industrial de uma rede de supermercados do Rio de Janeiro, e analisar a presença de genes codificadores da enterotoxina estafilocócica H (seh) e de resistência a antimicrobianos $(m e c A)$. Foram coletadas 50 amostras entre janeiro e março de 2016. Após o isolamento, foram realizadas a caracterização das amostras através de identificação em nível de espécie dos isolados por MALDI-TOF MS, a identificação dos genes seh e mecA, pelo método de reação em cadeia da polimerase (PCR). Das 50 amostras analisadas, 45 (90\%) foram positivas para presença de Staphylococcus sp. 41 amostras eram pertencentes ao gênero, 37 das quais foram identificadas em nível de espécie, sendo 40 amostras Staphylococcus coagulase-negativas (SCN) e uma coagulase-positiva (SCP). A identificação das espécies pela técnica de MALDI-TOF MS demonstrou ser acurada. O gene seh fora detectado em uma amostra de SCN (S. saprophyticus) isolada de fatiadora de frios. Quando realizada a técnica de PCR para a detecção do gene mecA nas 41 estirpes obtidas de Staphylococcus sp., 6 amostras de alimentos e 7 amostras de superfícies foram positivas. Conclui-se que os SCN se encontram disseminados no ambiente e precisam ter maior atenção no que diz respeito a sua detecção em alimentos.

Palavras-chave: Staphylococcus. Contaminação de Alimentos. Espectrometria de Massas. Resistência à Meticilina. Toxinas Bacterianas 


\section{Abstract}

Staphylococcus are microorganisms which are increasingly associated with food poisoning. This work aimed to detect and determine the prevalence of virulence and resistance factors from Staphylococcus spp. isolated from food, utensils and handling surfaces of an industrial kitchen of a supermarket chain in Rio de Janeiro, and analyze the presence of genes encoding staphylococcal enterotoxin $\mathrm{H}(\mathrm{seh})$ and antimicrobial resistance (mecA). 50 samples were collected between January and March. After isolation, microbial species were identified by MALDI-TOF MS, as well as detection of seh and mecA genes, by polymerase chain reaction (PCR). 45 of 50 samples analyzed (90\%), were positive for the Staphylococcus sp. presence. 41 samples were of the genus, where 37 were identified at species level, 40 samples coagulase-negative Staphylococcus (SCN), and one coagulasepositive (SCP). Identification of the species by the MALDI-TOF MS technique proved to be accurate. The seh gene was detected in only one CNS sample (S. saprophyticus), isolated from a meat slicer. When the PCR technique was performed to detect mecA gene in the 41 strains obtained from Staphylococcus sp., 6 food samples and 7 surface samples were positive. It is concluded that SCN are widespread in the environment and need more attention about its detection in food.

Keywords: Staphylococcus. Food Contamination. Mass Spectrometry. Methicilin-resistance. Bacterial Toxins.

\section{Introdução}

O gênero Staphylococcus compreende cocos gram-positivos, anaeróbios facultativos, imóveis e catalase positiva, ${ }^{1}$ sendo dividido em dois grupos com base na produção de coagulase, que são: Staphylococcus coagulase-positivos (SCP) e Staphylococcus coagulase-negativos (SCN). Dentro do grupo dos SCP, encontramos o $S$. aureus, patógeno mais descrito e com potencial virulento elevado, além de outros, como S. delphini, S. intermedius, S. coagulans e algumas cepas de $S$. hyicus. ${ }^{2}$ Entre os SCN, podem ser citados S. epidermidis, S. saprophyticus e espécies mais associadas a infecções, como $S$. haemolyticus e $S$. lugdunensis, tidos como principais patógenos nosocomiais. Os SCN têm sido considerados um grupo de risco para contaminação de alimentos, visto que são encontrados em larga escala em alimentos e, muitos deles, são capazes de produzir toxinas, causando intoxicação alimentar. ${ }^{3}$ 
A intoxicação alimentar estafilocócica é uma das doenças transmitidas por alimentos mais comuns no mundo e ocorre após a ingestão de Enterotoxinas Estafilocócicas (SE, Staphylococcal Enterotoxins), que são produzidas por estirpes enterotoxigênicas de Staphylococcus, principalmente $S$. aureus, e muito ocasionalmente por outras espécies de estafilococos, como S. intermedius. ${ }^{2}$ As enterotoxinas estafilocócicas são os principais agentes de intoxicação de origem bacteriana no homem e têm sido relatadas em vários surtos de doenças transmitidas por alimentos. As toxinas são termoestáveis ${ }^{4}$ ) e originam sintomas como vômito e diarreia, entre outros. ${ }^{5}$

A intoxicação alimentar causada pelas toxinas produzidas por Staphylococcus é um risco presente em alimentos manejados e armazenados de forma imprópria, porém, além das toxinas produzidas e seus efeitos adversos no organismo, há também o risco de os alimentos se tornarem reservatórios para micro-organismos com resistência a antibióticos. ${ }^{4}$

Os Staphylococcus aureus resistentes à meticilina, geralmente referido pelas siglas MRSA (do inglês Methicillin-resistant Staphylococcus aureus) ou ORSA (do inglês Oxacillin-resistant Staphylococcus aureus), são patógenos que se tornaram resistentes a diversos antimicrobianos primeiramente, à penicilina, em 1947, e, logo depois, à meticilina. ${ }^{6}$ Os sintomas de uma infecção por MRSA podem progredir substancialmente no período de 24-48 horas. ${ }^{7}$

A resistência à meticilina e outros antibióticos $\beta$-lactâmico sem estirpes de MRSA é ocasionada pela expressão de um gene contido no genoma destes, denominado mecA. ${ }^{7}$ Este gene está contido em um cassete cromossômico de 21 a 60 kb denominado SCCmec, um elemento genético móvel que pode também conter estruturas genéticas, como Tn554, pUB110, pT181. ${ }^{8}$ Após a aquisição do gene mecA, o mesmo deve ser integrado e localizado no cromossoma de $S$. aureus, e codifica uma proteína de ligação à penicilina alterada, denominada PBP2a, que difere de outras proteínas de ligação à penicilina. ${ }^{9}$ Segundo dados do International Working Group on the Staphylococcal Cassette Chromosome elements ${ }^{10}$ já foram descritos 11 tipos de SCCmec apresentando combinações diferentes de seis classes do complexo do gene mec (A, B, C1, C2, D, E) e de oito tipos de complexo $c c r .{ }^{9}$

Perante a possível ineficácia durante a etapa de higienização dos utensílios utilizados nos preparos dos alimentos e possíveis falhas durante a cocção dos alimentos e manutenção de temperaturas adequadas durante a etapa de porcionamento, faz-se necessário um estudo mais detalhado que possa identificar cepas de Staphylococcus sp. isolados dos alimentos e utensílios em questão.

Este trabalho teve como objetivo identificar cepas de Staphylococcus isolados de amostras de alimentos produzidos em uma cozinha industrial de uma rede de supermercados do Rio de Janeiro e dos seus respectivos utensílios e detectar genes associados à resistência a antimicrobianos (mecA) e produção de enterotoxinas (seh). 


\section{Metodologia}

Foram coletadas 50 amostras de alimentos, superfícies e utensílios utilizados em uma cozinha industrial de uma rede de supermercados do Rio de Janeiro, de janeiro a março de 2016. 21 amostras foram de alimentos e de origem proteica e 29 amostras foram provenientes dos utensílios e/ou superfícies usados para pré-preparo e preparo dos alimentos. Para a coleta dos alimentos, foram usados sacos esterilizados do tipo stomacher, e para pesquisa de contaminação dos utensílios utilizados nos preparos dos alimentos, empregou-se swabs estéreis para coleta. As amostras foram acondicionadas em bolsa térmica com blocos de gelo e transportadas até o Laboratório de Microbiologia, onde as análises foram realizadas.

Para isolamento de Staphylococcus em alimentos, 25 gramas do alimento em questão foram suspensos em $225 \mathrm{~mL}$ de água peptonada estéril, sendo posteriormente homogeneizados em stomacher. Após este procedimento, 0,1 mL da suspensão foi inoculada em ágar Baird-Parker (Himedia) para isolamento de colônias típicas de Staphylococcus ${ }^{11}$ Não foram realizados os plaqueamentos de diluições subsequentes, devido ao caráter qualitativo da análise. Foram levadas em conta as amostras com mais de 30 colônias típicas crescidas em placa (que acarreta o mínimo de $3,0 \times 10^{3} \mathrm{UFC} / \mathrm{g}$ ) como determinantes para a amostra ser considerada positiva e com no máximo 200 colônias, seguindo a Instrução Normativa $n^{\circ} 62 .{ }^{11}$

Para análise de superfícies e utensílios, após coleta de amostras utilizando swab estéril embebido em solução salina, estes foram semeados em Agar Manitol Salgado, realizando-se a técnica de esgotamento (método qualitativo), e após semeadura, estas foram incubadas a $36 \pm 2 \stackrel{\circ}{\mathrm{C}} / 18-24 \mathrm{~h}$ para posterior análise visual, onde as amostras com colônias características de estafilococos foram semeadas para placas contendo o TSA (Agar Triptona de Soja, Himedia) e incubadas em estufa a $36 \pm 2{ }^{\circ} \mathrm{C} / 18-24 \mathrm{~h}$ para crescimento e isolamento. As amostras foram estocadas em criotubos contendo 0,8 mL de TSB (Caldo Soja Triptona, Himedia) e 0,2 mL de Glicerina, a -20 ํ.

Conforme preconizado pela Instrução Normativa ${ }^{\circ}$ 62, que regulamenta os Métodos Analíticos Oficiais para Análises Microbiológicas para Controle de Produtos de Origem Animal e Água, ${ }^{11}$ para a caracterização do gênero Staphylococcus foram realizados os testes de coloração de Gram e prova da catalase. Após esta análise, foi aplicada a prova de coagulase, para identificar as cepas, como SCP ou SCN.

Para identificação das espécies de Staphylococcus isoladas, estas foram inoculadas em TSA por 24 h e transferidas para uma placa de 96 poços, para serem submetidas à Espectrometria de Massas (MALDI-TOF MS - Bruker, modelo Microflex LT), onde as amostras foram aplicadas em duplicata.

Para pesquisa dos genes mecA e seh, a liberação do DNA bacteriano foi feita por lise térmica. 
A detecção do gene seh, que codifica a enterotoxina estafilocócica $\mathrm{H}$, foi realizada através da técnica de PCR, conforme descrito por Sila e colaboladores, ${ }^{12}$ enquanto a detecção do gene mec $A$ foi realizada conforme descrito por Santos e colaboradores. ${ }^{13}$ Como controles, foram utilizadas as cepas de $S$. aureus ATCC 33591, positiva para o gene mecA e negativo para $\operatorname{seh}^{12}$ e S. aureus 633a, positiva para o gene seh e negativo para $m e c A .{ }^{11}$

Os produtos amplificados foram analisados por eletroforese em gel de agarose a 1,0\% em TBE (0,89 M Tris [Sigma], 0,89 M ácido bórico [Madison, WI, EUA], 2,5 mM EDTA [Sigma], pH 8,2), a $80 \mathrm{~V}$ por 1:30 h. O gel, previamente adicionado de $1 \mu \mathrm{L} / \mathrm{mL}$ de solução de GelRed ${ }^{\circledR}$, foi analisado sob luz ultravioleta. Como padrão de DNA para a corrida eletroforética, foi utilizado 100 pb DNA ladder (Biotools).

\section{Resultados e Discussão}

Das amostras isoladas de alimentos, 19 (90\%) foram positivas para Staphylococcus sp. As amostras isoladas de utensílios ou superfície foram semeadas em Agar Manitol Salgado, onde 26 amostras (89\%) foram positivas para Staphylococcus sp., sendo 3 destas fermentadoras de manitol. Das 45 amostras positivas para presença de Staphylococcus sp., duas eram catalase-negativas (identificadas pela técnica de MALDI-TOF MS como Enterococcus faecium e Enterococcus faecalis). Quatro amostras foram identificadas apenas em nível de gênero, sendo estas não pertencentes ao grupo Staphylococcus.

Das 41 amostras de Staphylococcus analisadas, 16 (39\%) foram identificadas como S. saprophyticus, $14(34 \%)$ como S. sciuri, 3 (7\%) como S. vitulinus, 2 (5\%) como S. xylosus, 1 (2\%) como S. carnosus, 1 (2\%) como S. aureus e 4 (11\%) como Staphylococcus sp. Apesar de não ser associado frequentemente a amostras de alimentos, percebe-se a alta incidência de $S$. saprophyticus, um SCN associado a infecções urinárias comunitárias, o que remete à contaminação proveniente de manipuladores, bem como S. sciuri, proveniente de microbiota de animais domésticos. ${ }^{13,14}$

Paim e colaboradores ${ }^{15}$ demonstraram que a metodologia de identificação de micro-organismos utilizando MALDI-TOF MS produziu uma identificação concordante em 440 (97,8\%) dos 450 isolados de cocos gram-positivos identificados pelo método fenotípico convencional. A identificação das espécies pela técnica de MALDI-TOF MS demonstrou ser acurada e rápida. Das 45 amostras, apenas $4(9 \%)$ foram identificadas somente em nível de gênero. A técnica identifica os microorganismos em um tempo muito curto (< 10 min.), com um mínimo de preparação das amostras e que apresenta baixo custo em termos de reagentes. Uma identificação bioquímica convencional oneraria 48 a 72 horas para ser realizada, ou utilizaria kits de identificação comerciais que têm custo elevado. ${ }^{16}$ Além disso, é uma técnica que apresenta alta precisão em nível de espécies e que neste estudo apresentou $90 \%$ de precisão para identificação das espécies com confiabilidade para confirmação do gênero Staphylococcus. 
Das 41 amostras de Staphylococcus sp., 1 (2,4\%) era SCP (identificado como S. aureus por MALDI-TOF MS) e 40 (97,6\%), SCN. A amostra de S. aureus encontrada fora isolada de amostra de alimento (amostra isolada de asa de frango), e não foram detectados nesta amostra os genes seh e mecA. No entanto, não foram encontradas amostras positivas de SCP isoladas de superfícies e utensílios, enquanto Silva ${ }^{17}$ (2006) obteve altas contagens de SCP em estudo de avaliação da qualidade microbiológica de utensílios e superfícies de manipulação de alimentos de unidade de alimentação. O grande percentual de amostras positivas de Staphylococcus spp. em superfícies de preparo de alimentos demonstra a importância desses locais como fontes potenciais de disseminação de micro-organismos, principalmente através da contaminação cruzada de alimentos. Cabe ressaltar que a contaminação cruzada tem sido frequentemente relatada como fator responsável pela ocorrência de enfermidades de origem alimentar. ${ }^{18}$ No entanto, percebe-se que cada vez mais os SCN vêm sido descritos associados a amostras relacionadas a alimentos. Em um estudo recente de Santos, ${ }^{19}$ de um total de 60 amostras isoladas de superfície em uma indústria de laticínios, 41 pertenciam ao gênero Staphylococcus, sendo 16 (39,0\%) destas SCP e 25 (61,0\%), SCN, mostrando que tais achados são preocupantes principalmente se considerada a capacidade enterotoxigênica de algumas estirpes de estafilococos coagulase-positivo e estafilococos coagulase-negativo, fato este que pode significar a ocorrência de riscos para a saúde pública. Em nosso estudo, observou-se também uma taxa elevada de isolamento de SCN.

Quando realizada a técnica de PCR para a detecção do gene mecA nas 41 estirpes obtidas de Staphylococcus sp., 6 amostras de alimentos e 7 amostras de superfícies foram positivas para o gene (Tabela 1). As espécies portadoras dos genes estão relacionadas também na Tabela 1. Encontramos neste estudo amostras de $S$. saprophyticus, S. sciuri, S. vitulinus e $S$. xylosus portadoras do gene mecA. Amostras destes SCN multirresistentes já vêm sido descritas na literatura. ${ }^{20,21,22}$ Para confirmação da expressão do gene $m e c A$, que codifica resistência à oxacilina e outros antimicrobianos, as amostras foram submetidas à triagem em ágar contendo $4 \mu \mathrm{g} / \mathrm{mL}$ de oxacilina, conforme Ferreira e colaboradores. ${ }^{23}$ Todas as amostras cresceram confluentemente, mostrando que todas expressavam o gene. O gene mecA encontra-se altamente conservado entre as espécies de Staphylococcus e sua origem ainda não está definida, porém alguns estudos demonstram que há similaridade entre as sequências de aminoácidos das PBPs de $S$. sciuri e de $S$. aureus, o que sugere que o gene mecA pode ter se originado a partir de Staphylococcus coagulase-negativos. ${ }^{24}$ 
Tabela 1. Características referentes às 41 estirpes obtidas de Staphylococcus sp. encontradas nas amostras de alimentos, superfície e utensílios coletados em uma rede de supermercados do Estado do Rio de Janeiro.

\begin{tabular}{cccc}
\hline Amostras & Origem & mecA & seh \\
\hline SCP (1) & Alimento (1) & Positivo (0) & Positivo (0) \\
\hline & & Staphylococcus sp. (2) & \\
& Alimento (13) & S. saprophyticus (2) & Positivo (0) \\
& & S. vitulinus (1) & \\
SCN (40) & S. xylosus (1) & \\
\cline { 2 - 4 } & & Staphylococcus sp. (2) & S. saprophyticus (1)* \\
& Superfície / & S. saprophyticus (3) & \\
& Utensílio (27) & S. vitulinus (1) & \\
& S. sciuri (1) & \\
& &
\end{tabular}

*Amostra isolada de fatiadora de frios.

Os SCN apresentaram um porcentual expressivo (32\%) para positividade do gene mecA, principalmente nas amostras de superfícies, e este tipo de gene pode ser transferido entre espécies do gênero Staphylococcus e favorecer a contaminação cruzada entre superfície x manipulador x alimento, o que poderá contribuir para a contaminação alimentar e a possibilidade de ocorrência de DTAs.

O gene seh fora detectado em uma amostra de SCN (S. saprophyticus) isolada de fatiadora de frios. Este resultado é importante, pois a Legislação Brasileira não recomenda a pesquisa de SCN (apenas a detecção de coagulase), de forma que esta amostra toxigênica não seria detectada se apenas os parâmetros atuais fossem utilizados. Para fins de confirmação, os amplicons de 336 pb encontrados na amostra controle (633a) e da amostra isolada no presente estudo foram sequenciados e confirmados como pertencentes a um fragmento do referido gene. Durante muito tempo, o $S$. aureus foi considerado a única espécie patogênica entre as espécies de Staphylococcus, enquanto os SCN eram classificados como agentes contaminantes. No entanto, atualmente, as técnicas de biologia molecular têm mostrado que estes micro-organismos também possuem genes que codificam enterotoxinas e outros fatores de virulência. ${ }^{25}$ Borges e colaboradores ${ }^{26}$ recomendaram que a presença de espécies de SCN não seja ignorada em investigações de casos suspeitos de intoxicação estafilocócica, uma vez que este grupo de patógenos, estando presente no alimento, oferece risco de causar intoxicação ao consumidor. 
A produção de coagulase é uma característica utilizada na identificação de $S$. aureus. ${ }^{27}$ A legislação brasileira, através da Resolução RDC n $12,{ }^{28}$ indica a pesquisa de estafilococos coagulasepositiva como indicativo de $S$. aureus. Apesar de a legislação brasileira não exigir a pesquisa da presença de (SCN), estudos mostram que estes são produtores de enterotoxinas assim como os SCP, em especial $S$. aureus, associado com surtos de doenças transmitidas por alimentos. ${ }^{29}$ No presente estudo, apenas uma amostra apresentava enterotoxina H e esta era SCN. Devido a esse fato, os mesmos têm recebido mais atenção pelos governos e indústrias ao estabelecer normas, métodos ou especificações microbiológicas. ${ }^{30}$ Sendo assim, sugere-se que a legislação brasileira inclua a pesquisa de SCN como uma nova categoria de avaliação. ${ }^{28}$

\section{Conclusão}

Conclui-se que, de acordo com os resultados encontrados no presente estudo, nota-se um alto percentual de contaminação dos produtos alimentícios e principalmente das superfícies que entram em contato direto com os alimentos, sugerindo uma deficiência do ponto de vista higiênico-sanitário, sendo necessária a implementação de técnicas que possam diminuir ou sanar a ocorrência da multiplicação bacteriana nos alimentos e superfícies. O número expressivo de estirpes SCN reforça o crescimento dos mesmos em casos de contaminação alimentar e é sugestivo para que a legislação brasileira inclua a pesquisa de SCN como uma nova categoria de avaliação

\section{Colaboradores}

PL Nascimento trabalhou em todas as etapas, desde a concepção do estudo até a revisão da versão final do artigo. JPO Martinez contribuiu na etapa de MALDI-TOF e PCR e na conclusão final do trabalho.

Conflito de Interesses: Os autores declaram não haver conflito de interesses.

\section{Referências}

1. Becker K, Von Eiff C. Staphylococcus, micrococcus, and other catalase-positive cocci. In: Becker K, Robert LS, Von Eiff C. Manual of clinical microbiology. 11 ed. Washington, DC: American Society of Microbiology Press; 2015. p. 308-309.

2. Hennekinne JA, Ostyn A, Guillier F, Herbin S, Prufer AL, Dragacci S, et al. How should staphylococcal food poisoning outbreaks be characterized? Toxins 2011; 2(8):2106-2116.

3. Nunes R SC, Del-Aguila E, Paschoalin V. Safety evaluation of the coagulase-negative staphylococci microbiota of salami: superantigenic toxin production and antimicrobial resistance. Biomed Res Int. 2015; 2015:1-17. 
4. Regenthal, P, Hansen JS, André I, Lindkvist-Petersson K. Thermal stability and structural changes in bacterial toxins responsible for food poisoning. Plos One 2017; 12(2):1-15.

5. Muratovic AZ, Hagström T, Rosén J, Granelli K, Hellenäs KE. Quantitative analysis of Staphylococcal enterotoxins $\mathrm{A}$ and $\mathrm{B}$ in food matrices using ultra high-performance liquid chromatography tandem mass spectrometry (UPLC-MS/MS). Toxins 2015; 7(9):3637-3656.

6. Grunther RA. MRSA: Multiresistencer Staphylococcus aureus. Berlin: Medizinisch Orthopadische Technik; 2011.

7. Hussein NR, Basharat Z, Muhammed AH, Al-Dabbagh SA. Comparative evaluation of MRSA nasal colonization epidemiology in the urban and rural secondary school community of Kurdistan, Iraq. Plos One 2015; 10(5):1-9.

8. Rolo J, Worning P, Nielsen JB, Bowden R, Bouchami O, Damborg P, et al. Evolutionary origin of the staphylococcal cassette chromosome mec (SCCmec). Antimicrobial Agents and Chemotherapy 2017; 61(5):1-38.

9. Long SW, Olsen RJ, Mehta SC, Palzkill T, Cernoch PL, Perez KK, et al. PBP2a mutations causing high-level ceftaroline resistance in clinical methicillin-resistant Staphylococcus aureus isolates. Antimicrob Agents Chemother. 2014; 58(11):6668-6674.

10. IWG-SCC [Internet]. International Working Group on the Staphylococcal Cassette Chromosome elements. [acesso em: 07 set 2016]. Disponível em: http://www.sccmec.org/Pages/SCC_HomeEN.html

11. Brasil. Instrução Normativa no 62, de 26 de agosto de 2003. Oficializa os Métodos Analíticos Oficiais para Análises Microbiológicas para Controle de Produtos de Origem Animal e Água. Diário Oficial da União 18 set. 2003.

12. Sila J, Sauer P, Kolar M. Comparison of the prevalence of genes coding for enterotoxins, exfoliatins, panton-valentine leukocidin and tsst-1 between methicillin-resistant and methicillin-susceptible isolates of Staphylococcus aureus at the University Hospital in Olomouc. Biomed Pap Med Fac Univ Palacky Olomouc Czech Repub 2009; 153(3):215-218.

13. Santos KRN, Teixeira LM, Leal GS, Fonseca LS, Gontijo-Filho PP. DNA typing of methicillinresistant Staphylococcus aureus: isolates and factors associated with nosocomial acquisition in two Brazilian university hospitals. J Med Microbiol. 1999; 48(1):17-23.

14. Bannerman TL, Peacock SJ. Staphylococcus, micrococcus and other catalase-positive cocci that grow aerobically. In: Murray PR, Barron EJ, Pfaller MA, Tenover FC, Yolken RH. Manual of clinical microbiology. 9. ed. Washington DC: ASM Press; 2007. p. 390-411.

15. Paim T, Reiter K, Oliveira C, Azevedo P. Desempenho da metodologia MALDI-TOF MS na identificação de cocos Gram-positivos na cidade de Porto Alegre/RS, Brasil. Journal of Infection Control 2013; 2(2):112-16.

16. Santos AF, Cayô R, Schandert L, Gales AC. Avaliação da técnica de MALDI-TOF MS no laboratório de Microbiologia. J Bras Brasil Patol Med Lab. 2013; 49(3):191-97.

17. Silva LF. Procedimento operacional padronizado de higienização como requisito para segurança alimentar em unidade de alimentação [Dissertação]. [Santa Maria]: Universidade Federal de Santa Maria; 2006. 
18. Silva EA. Manual de controle higiênico-sanitário em alimentos. 5. ed. São Paulo: Varela; 2002.

19. Santos S. Investigação da presença e da formação de biofilmes por Estafilococos em micro-usina de beneficiamento de leite [Dissertação]. São Paulo: Universidade Estadual Paulista Júlio de Mesquita; 2009.

20. Barghava K, Zhang Y. Characterization of methicillin-resistant coagulase-negative staphylococci (MRCoNS) in retail meat. Food Microbiol. 2014; 42:56-60.

21. Kürekci C. Short communication: Prevalence, antimicrobial resistance, and resistant traits of coagulasenegative staphylococci isolated from cheese samples in Turkey. J Dairy Sci. 2016; 99(4):2675-9.

22. Jeong DW, Lee B, Her JY, Lee KG, Lee JH. Safety and technological characterization of coagulasenegative staphylococci isolates from traditional Korean fermented soybean foods for starter development. Int J Food Microbiol. 2016; 236:9-16.

23. Ferreira RB, Iorio NL, Malvar KL, Fonseca LS, Bastos CC, Santos KR. Coagulase-negative staphylococci: comparison of phenotype and genotypic oxacillin susceptibility tests and evaluation of the agar screening test by using different concentrations of oxacillin. J Clin Microbiol. 2003; 41(8):3609-3614.

24. Wu S, Piscitelli C, Lencastre H, Tomasz A. Tracking the evolutionary origin of the methicillin resistance gene: cloning and sequencing of a homologue of mecA from a methicillin susceptible strain of Staphylococcus sciuri. Microb Drug Resist. 1996; 2(4):435-41.

25. Rall VLM, Sforcin JM, Deus MFR, Sousa DC, Camargo CH, Godinho NC. Polymerase Chain Reaction Detection of Enterotoxins Genes in Coagulase-Negative Staphylococci Isolated from Brazilian Minas Cheese. Foodborne Pathog Dis. 2010; 7(9):1121-23.

26. Borges MDF, Nassu RT, Pereira JL, Andrade APCD, Kuaye AY. Perfil de contaminação por Staphylococcus e suas enterotoxinas e monitorização das condições de higiene em uma linha de produção de queijo de coalho. Ciênc Rural 2008; 38(5):1431-38.

27. Silva ER, Silva N. Coagulase gene typing of Staphylococcus aureus isolated from cows with mastitis in southeastern Brazil. Can J Vet Res. 2005; 69(4):260-264.

28. Brasil. Agência Nacional de Vigilância Sanitária. Resolução nº 12, de 02 de janeiro de 2001. Aprova o Regulamento Técnico sobre padrões microbiológicos para alimentos. Diário Oficial da União 02 jan 2001; Seção 1.

29. Veras JF, Carmo LS, Tong LC, Shupp JW, Cummings C, Santos D, et al. A study of the enterotoxigenicity of coagulase-negative and coagulase-positive staphylococcal isolates from food poisoning outbreaks in Minas Gerais, Brazil. Int J Infect Dis. 2008; 12(4):410-15.

30. Zell C, Resch M, Rosenstein R, Albrecht T, Hertel C, Götz F. Characterization of toxin production of coagulase-negative staphylococci isolated from food and starter cultures. Int J Food Microbiol. 2008; 127(3):246-51.

Recebido: 22/11/2016

Revisado: 25/04/2017

Aceito: 16/05/2017 